\title{
Is there an Increase in Valgus Deviation in Tibial Distraction Using the Lengthening Over Nail Technique?
}

\author{
Hoon Park MD, Keun Jung Ryu MD, Hyun Woo Kim MD, PhD, \\ Jin Ho Hwang MD, PhD, Joon Woo Han MD, Dong Hoon Lee MD, PhD
}

Received: 29 July 2015/ Accepted: 12 January 2016/Published online: 29 January 2016

(C) The Association of Bone and Joint Surgeons $\mathbb{R} 2016$

\begin{abstract}
Background During tibial lengthening, the soft tissues of the posterolateral compartment produce distraction-resisting forces causing valgus angulation. Although this occurs with the classic Ilizarov method, whether a valgus deformity develops with the lengthening over nail (LON) technique is questioned, because the intramedullary nail is thought to resist deforming forces and adequately maintain alignment of the distracted bone.

Questions/purposes The purposes of this study were to (1) determine the amount of valgus deviation during tibial

Each author certifies that he or she, or a member of his or her immediate family, has no funding or commercial associations (eg, consultancies, stock ownership, equity interest, patent/licensing arrangements, etc) that might pose a conflict of interest in connection with the submitted article.

All ICMJE Conflict of Interest Forms for authors and Clinical Orthopaedics and Related Research ${ }^{\mathbb{R}}$ editors and board members are on file with the publication and can be viewed on request.

Clinical Orthopaedics and Related Research ${ }^{\mathbb{B}}$ neither advocates nor endorses the use of any treatment, drug, or device. Readers are encouraged to always seek additional information, including FDAapproval status, of any drug or device prior to clinical use. Each author certifies that his or her institution approved the human protocol for this investigation, that all investigations were conducted in conformity with ethical principles of research, and that the informed consent for participation in the study was obtained. This study was performed at Severance Children's Hospital, Seoul, Korea.
\end{abstract}

\section{H. Park}

Department of Orthopedic Surgery, Gangnam Severance Hospital, Yonsei University College of Medicine, Seoul, Korea

K. J. Ryu, H. W. Kim, J. H. Hwang, J. W. Han, D. H. Lee ( $\square)$ Division of Orthopedic Surgery, Severance Children's Hospital, Yonsei University College of Medicine, Seoul 03722, Korea e-mail: orthopaedee@naver.com; drdonghoon@yuhs.ac lengthening with the LON technique; and (2) analyze the factors that may be associated with valgus deviation with the LON technique.

Methods Between June 2009 and September 2013, we performed 346 tibial lengthenings using the LON technique, lengthening and then nail technique, or lengthening with an intramedullary lengthening device. Sixty patients (120 tibias) who underwent bilateral lower leg lengthening with the LON technique were enrolled in this retrospective study. To limit the number of variables, we analyzed only the right tibia in all patients (60 tibias). The mean followup was 42 months (range, 26-71 months). The mean age of the patients was 25 years (range, 18-40 years). There were 36 male and 24 female patients. The mean final length gain was $67 \pm 9 \mathrm{~mm}$. The mean time for distraction was $100 \pm 25$ days. The overall valgus deviation was assessed by measuring the change in the medial proximal tibial angle and mechanical femorotibial angle on radiographs obtained before and after surgery and after completion of lengthening. Several demographic, surgical, and distraction-related variables were considered possible factors to prevent valgus deviation: proximal fixation method; presence of a blocking screw; diameter and length of the intramedullary nail; degree of nail insertion; length of the nail in the distal segment after completion of distraction; final length gain; and patient's BMI. During the period studied, the blocking screw was to maintain the mechanical axis in patients who had neutral or valgus alignment preoperatively, or to prevent more valgus change in patients who underwent acute correction of varus deformity intraoperatively. Uni- and multivariate analyses were conducted.

Results Valgus deviation occurred during the tibial LON. The medial proximal tibial angle increased from $86^{\circ}(95 \%$ $\left.\mathrm{CI}, 85^{\circ}-86^{\circ}\right)$ to $90^{\circ}\left(95 \% \mathrm{CI}, 89^{\circ}-91^{\circ}\right)(\mathrm{p}<0.001)$. The mechanical femorotibial angle changed from $2.2^{\circ}$ varus $\left(95 \% \mathrm{CI}, 3^{\circ}-1.4^{\circ}\right.$ varus) to $2.6^{\circ}$ valgus $\left(95 \% \mathrm{CI}, 1.8^{\circ}-3.4^{\circ}\right.$ 
valgus) ( $\mathrm{p}<0.001)$. Valgus deviation was evident in proximal and distal segments. In the multivariate regression model, use of a blocking screw was the only factor that was associated with decreased valgus deviation, and its effect size, although detectable, was small $(-2.62 ; 95 \%$ CI, -4.65 to $-0.59 ; \mathrm{p}=0.013$ ).

Conclusions We found that valgus deviation does occur during tibial lengthening using the LON technique, but that blocking screw placement may help to minimize the likelihood that severe valgus deviation will occur. Future prospective studies should be conducted to confirm this preliminary finding. Level of Evidence Level III, therapeutic study.

\section{Introduction}

The lengthening over an intramedullary nail (LON) technique was first introduced by Herzenberg and Paley in 1997 [5]. Despite the potential risk for deep infection [7, 9, 18], it has gained popularity because it enables more comfortable lengthening, shortens the period of external fixation, and provides a more stable construct of the regenerate bone supported internally with the intramedullary nail [6].

Another potential advantage of this technique is that it prevents the secondary deformity that can occur during distraction [3, 15, 20, 21]. In contrast to the classic Ilizarov method, the intramedullary nail in the LON technique is thought to resist distraction-resisting deforming forces [14, 20]. It can act as a guide such that the bone is lengthened along the longitudinal axis of the nail with no axial deviations. It was reported that the secondary deformity induced during distraction could be substantively negated with the intramedullary nail in the LON technique of the tibia [3, 15, 20, 21]. However, Kim et al. [8] reported that a valgus deviation was the most common complication in 20 segments $(25 \%)$ in their series of the LON technique of the tibia. Valgus deviation during tibial lengthening with the LON technique remains debated; to our knowledge, its quantitative assessment has not been documented.

The purposes of this study were to (1) determine the amount of valgus deviation during tibial lengthening with the LON technique; and (2) analyze the factors that may be associated with valgus deviation with the LON technique.

\section{Patients and Methods}

Patient Selection

This is a retrospective study that was approved by the institutional review board at our institution (BD2013-
024D). Between June 2009 and September 2013, we performed 346 tibial lengthenings using the LON technique, lengthening and then nail technique, or lengthening with an intramedullary lengthening device. During this period, one hundred 24 tibias in 62 patients who underwent bilateral lower leg lengthening for familial short stature were identified. Each limb lengthening surgery was performed with the LON technique. Inclusion criteria were: (1) skeletally mature patient; (2) no history of medical illness, fracture, soft tissue compromise, bony deformities, or infections of the lower extremity; (3) bilateral tibias with a similar amount of lengthening; and (4) availability of standing lower limb coronal orthoroentgenograms taken pre- and postoperatively. Two patients were excluded owing to insufficient radiographic evaluations. Sixty patients with bilateral tibial lengthening met the inclusion criteria. To limit the number of variables, we analyzed only the right tibia in all patients. Therefore, sixty patients $(60$ tibias) were enrolled in this study. No patients were lost to followup. The mean followup was 42 months (range, 2671 months). There was no preoperative deformity that did not allow the LON technique, such as narrow canal or severe angular deformity. The mean $( \pm$ SD) age of the patients was $24 \pm 5$ years (range, $18-40$ years). There were 36 male and 24 female patients. The mean $( \pm$ SD) preoperative height and final length gain were $161 \pm 7 \mathrm{~cm}$ and $67 \pm 9 \mathrm{~mm}$, respectively (Table 1 ).

\section{Surgical Procedure and Postoperative Rehabilitation}

All surgical procedures were performed by the senior author (DHL). The operative procedure was similar to the

Table 1. Demographic features of the 60 patients

Total 60

segments

\begin{tabular}{ll}
\hline Demographic variables & \\
Age (years) & $25 \pm 5$ \\
Sex (male:female) & $36: 24$ \\
Preoperative height $(\mathrm{cm})$ & $160 \pm 7$ \\
BMI $\left(\mathrm{kg} / \mathrm{m}^{2}\right)$ & $21 \pm 2$ \\
Duration of followup (months) & $42 \pm 16$ \\
Lengthening variables & \\
Final length gain (mm) & $67 \pm 9$ \\
Distraction rate (mm/day) & $0.78 \pm 0.12$ \\
External fixator index (months/cm) & $0.55 \pm 0.09$ \\
Intramedullary nail diameter (diameter; & $8 \mathrm{~mm} ; 28$ \\
number of segments) & $9 \mathrm{~mm} ; 17$ \\
& $10 \mathrm{~mm} ; 15$ \\
Intramedullary nail length (mm) & $295 \pm 17$ \\
\hline
\end{tabular}

Values are expressed as mean $\pm \mathrm{SD}$. 


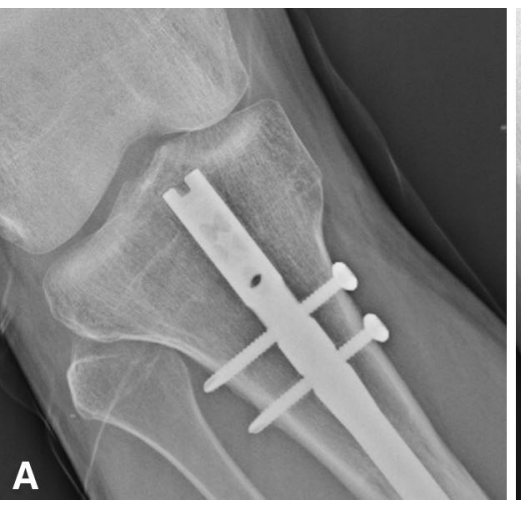

Fig. 1A-C A plain AP radiograph of the proximal tibia shows the three proximal fixation methods: (A) two standard mediolateral locking screws, (B) two mediolateral ASLS (angular stable locking

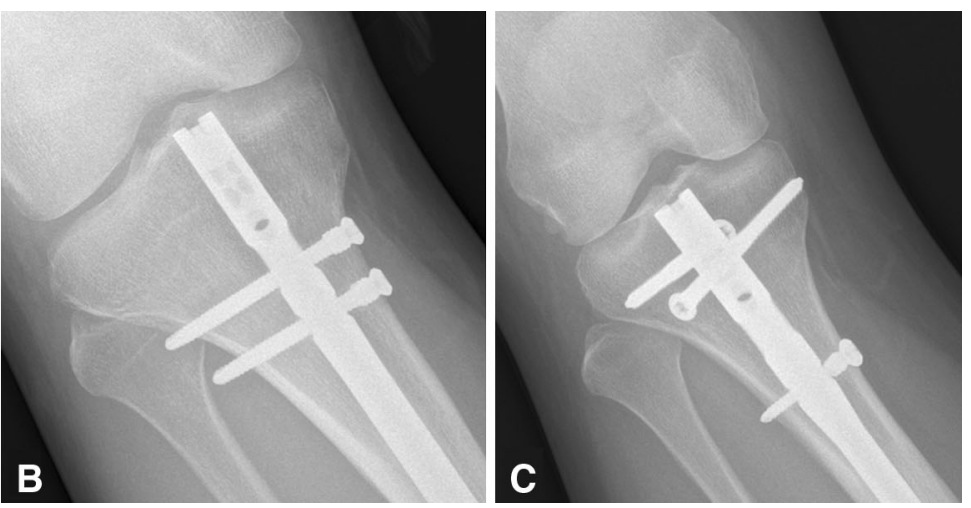

system) screws, and (C) one ASLS screw combined with two oblique cancellous bone screws.
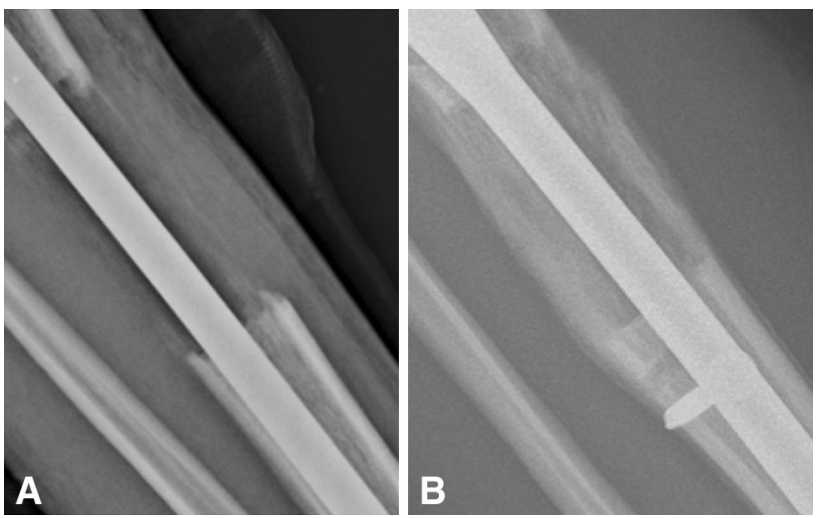

Fig. 2A-B Plain AP radiographs of the tibial shaft show (A) LON without a blocking screw and (B) with a blocking screw. The screw was inserted just below the level of the osteotomy and just lateral to the intramedullary nail in 28 of 60 patients. intramedullary nail and the screw; or (3) one ASLS screw combined with two oblique cancellous bone screws (Fig. 1). The surgeon switched the fixation method of the proximal segment during the study period. Two standard locking screws were used in eight patients between June 2009 and May 2010. Two ASLS screws were used in 42 patients between June 2010 and August 2012, and the third method was used in 10 patients from September 2012 to September 2013. The choice of method was not randomized. A blocking screw was inserted just below the osteotomy level and just lateral to the intramedullary nail at the distal segment in an AP direction for 28 selected patients intraoperatively after insertion of the nail (Fig. 2). A blocking screw was used to maintain the mechanical axis of both tibias in patients who had neutral or valgus alignment preoperatively, or to prevent more valgus deviation of one or both tibias in patients who underwent acute correction of the varus deformity intraoperatively. After placement of the blocking screw, we checked the alignment of the entire tibia under an image intensifier. The two groups (one group with a blocking screw and one without a blocking screw) were comparable in terms of preoperative variables and final length gain (Table 2). The Ilizarov ring fixators (S.H. Pikar Orthotools Pvt, Pune, India) were mounted.

The latent period was 7 to 9 days for all segments. The distraction rate was set to be $1 \mathrm{~mm}$ per day but was adjusted depending on the amount of callus increment based on weekly radiographic evaluations for inpatients or every 2 weeks for outpatients. Postoperatively, all patients were encouraged to do ROM exercises of the knees and ankles on a daily basis under the guidance of a registered physical therapist (JHP). After completion of the distraction period, two distal interlocking screws were inserted and the external fixator was removed. All patients were allowed to do full weightbearing when two cortical bridges of the tibia were identified on the radiograph. Patients were followed up biweekly during the distraction phase and monthly during 
Table 2. Comparison of demographic features between the patients who received a blocking screw and those who did not

\begin{tabular}{|c|c|c|c|}
\hline Variables & $\begin{array}{l}\text { Blocking screw } \\
(\mathrm{n}=28)\end{array}$ & $\begin{array}{l}\text { No blocking screw } \\
(\mathrm{n}=32)\end{array}$ & $\begin{array}{l}\mathrm{p} \\
\text { value }\end{array}$ \\
\hline Age (years) & $24 \pm 5$ & $24 \pm 6$ & 0.998 \\
\hline Sex (male:female) & $19: 9$ & $17: 15$ & 0.245 \\
\hline $\begin{array}{l}\text { Preoperative height } \\
\text { (cm) }\end{array}$ & $160 \pm 8$ & $162 \pm 6$ & 0.302 \\
\hline BMI $\left(\mathrm{kg} / \mathrm{m}^{2}\right)$ & $21 \pm 2$ & $21 \pm 2$ & 0.764 \\
\hline $\begin{array}{l}\text { Duration of followup } \\
\text { (months) }\end{array}$ & $38 \pm 15$ & $46 \pm 16$ & 0.105 \\
\hline Nail diameter (mm) & $9 \pm 1$ & $9 \pm 1$ & 0.538 \\
\hline Nail length (mm) & $298 \pm 20$ & $293 \pm 15$ & 0.233 \\
\hline $\begin{array}{l}\text { Preoperative MPTA } \\
\text { (degrees) }\end{array}$ & $85 \pm 2$ & $86 \pm 2$ & 0.123 \\
\hline $\begin{array}{l}\text { Preoperative mFTA } \\
\text { (degrees) }\end{array}$ & $1.5 \pm 2.7$ varus & $2.8 \pm 2.3$ varus & 0.257 \\
\hline
\end{tabular}

Values expressed as mean \pm SD; MPTA $=$ medial proximal tibial angle $;$ mFTA $=$ mechanical femorotibial angle .

the consolidation phase. We routinely removed the nails approximately 2 years after the index operation.

\section{Evaluation of Valgus Deviation}

The overall valgus deviation was assessed by measuring the medial proximal tibial angle (MPTA) (Fig. 3) and mechanical femorotibial angle (mFTA) (a positive value indicates genu valgus, whereas a negative value indicates genu varum) (Fig. 4) from an AP plain radiograph of the tibia and from a standing lower limb coronal orthoroentgenogram, respectively, which were obtained preoperatively, postoperatively, and at final followup (after completion of lengthening). The postoperative mFTA was thought to be inadequate for evaluation because the patients wearing bilateral Ilizarov fixators could not stand in a proper position to take a standing lower limb orthoroentgenogram. The mechanical axis of the femur passes from the center of the hip through the center of the knee. The mechanical axis of the tibia passes from the center of the tibial spine through the center of the ankle mortise. The angle between these two axes is defined as the mFTA. Pairwise comparisons were made to see if the valgus deviations were significantly changed. Valgus deviation at the proximal segment was assessed by measuring the distance (Distance D) between the lateral margin of the intramedullary nail and the inner margin of the lateral cortex at proximal fragment (Fig. 5). Valgus deviation at the distal segment was assessed by measuring the angle (Angle A) between the longitudinal axis of the intramedullary nail and the longitudinal axis of the distal segment (Fig. 6). Distance $\mathrm{D}$ and Angle A were measured from the immediate

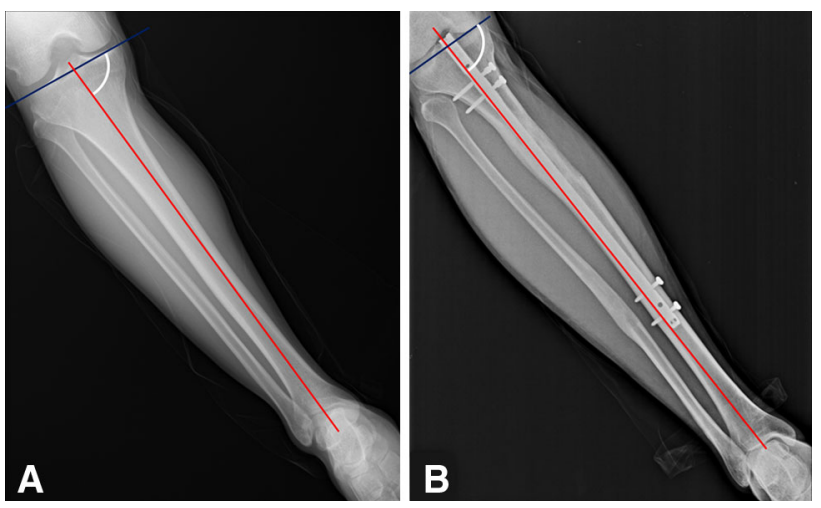

Fig. 3A-B Plain AP radiographs of the tibia show the medial proximal tibial angle (MPTA) (A) preoperatively and (B) at final followup. The red line indicates the midpoint of the knee and the ankle and the blue line indicates the proximal tibial joint line.

postoperative AP plain radiographs and after completion of distraction. Pairwise comparisons were made to see if the valgus deviations at the proximal and distal segments were significantly changed.

\section{Possible Factors Associated With Valgus Deviation}

Several demographic, surgical, and distraction-related variables were considered possible factors that could be related to unwanted valgus deviation. These were (1) the proximal fixation method between the intramedullary nail and the proximal fragment (two standard mediolateral locking screws, two ASLS screws, or one ASLS screw combined with two oblique cancellous bone screws); (2) presence of a blocking screw placed at the distal fragment (no or yes); (3) diameter $(\mathrm{mm})$ and length $(\mathrm{mm})$ of the intramedullary nail used; (4) depth of intramedullary nail insertion, which was measured as the distance from the distal tip of the nail to the tibial plafond on the immediate postoperative lateral plain radiograph (mm) (Fig. 7); (5) length of the intramedullary nail in the distal segment after completion of distraction (mm) (Fig. 8); (6) final length gain $(\mathrm{mm})$; and (7) BMI $\left(\mathrm{kg} / \mathrm{m}^{2}\right)$.

A correlation analysis was applied to determine if the overall valgus deviation had a significant correlation with factors 1 through 7 above. These possible factors were analyzed using univariate and multivariate statistical methods. It was postulated that use of ASLS screws in proximal fixation, use of a blocking screw, use of a thicker and longer intramedullary nail, deeper insertion of the intramedullary nail during surgery, longer length of the nail residing in the distal fragment after completion of lengthening, shorter final length gain, and lower BMI were related to less valgus deviation. 

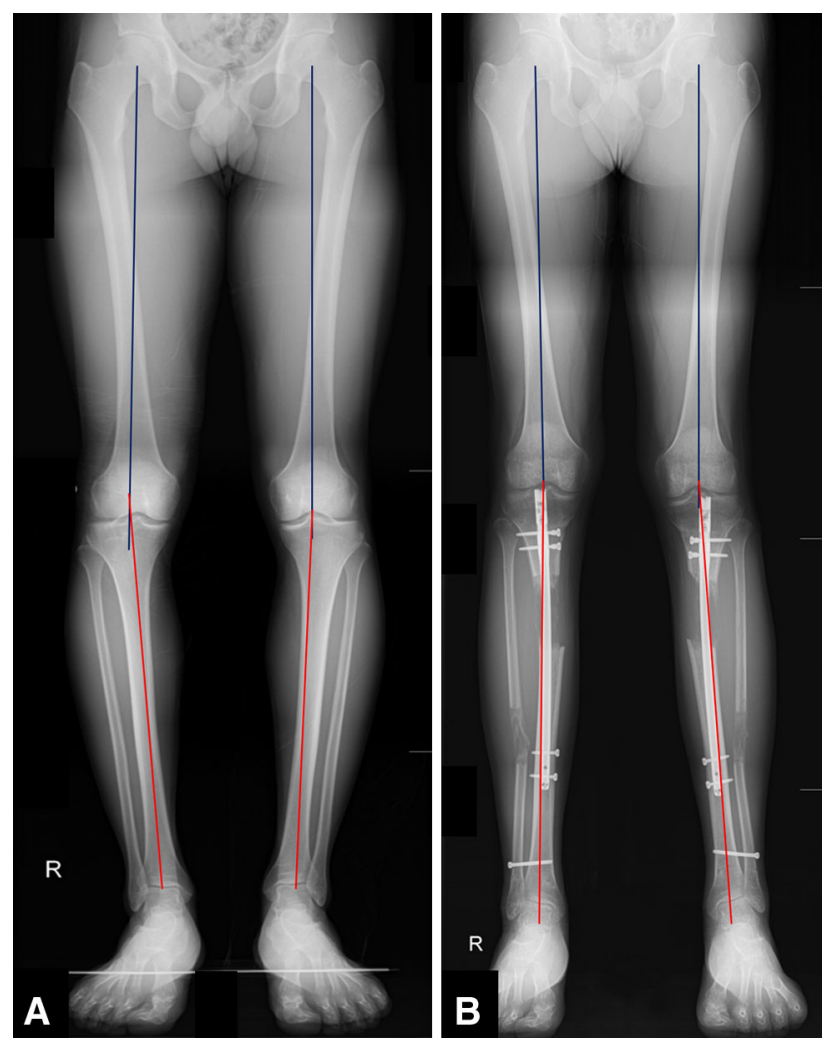

Fig. 4A-B Standing lower limb coronal orthoroentgenograms obtained (A) preoperatively and (B) at final followup show the mechanical femorotibial angle (mFTA). This is the angle between the mechanical axis of the femur (blue line) and the mechanical axis of the tibia (red line).
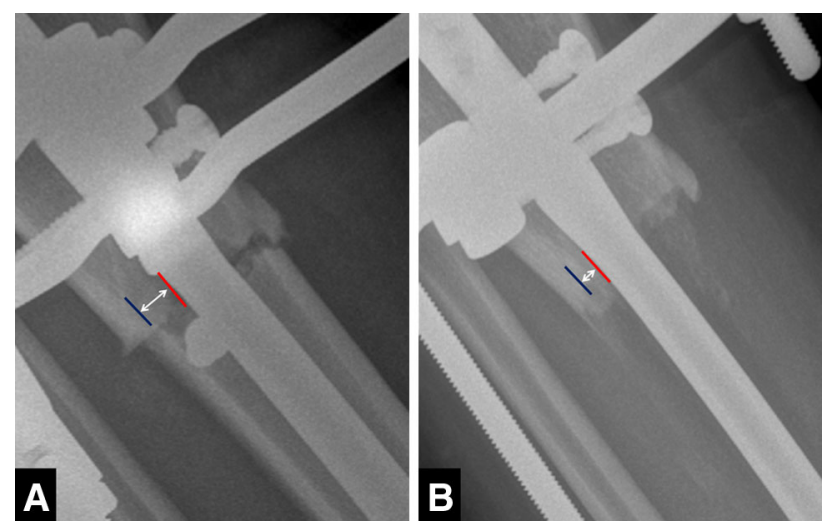

Fig. 5A-B Plain AP radiographs of the tibia around the osteotomy, taken (A) immediately postoperatively and (B) after completion of distraction site, show Distance D (white arrow), which is the distance between the lateral margin of the intramedullary nail (red line) and inner margin of the lateral cortex at the proximal fragment (blue line).

\section{Statistical Analysis}

All continuous variables were tested for normality using the Shapiro-Wilk test, which allowed normal distribution assumption. These were expressed as mean with a SD. A
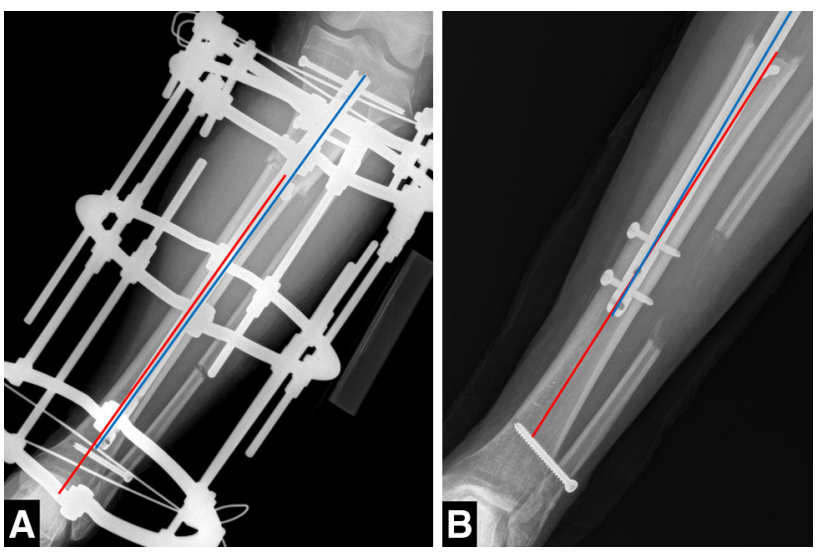

Fig. 6A-B Plain AP radiographs of the tibia taken (A) immediately postoperatively and (B) after completion of distraction show Angle A, which is the angle between the longitudinal axis of the intramedullary nail (blue line) and the longitudinal axis of the distal segment (red line).

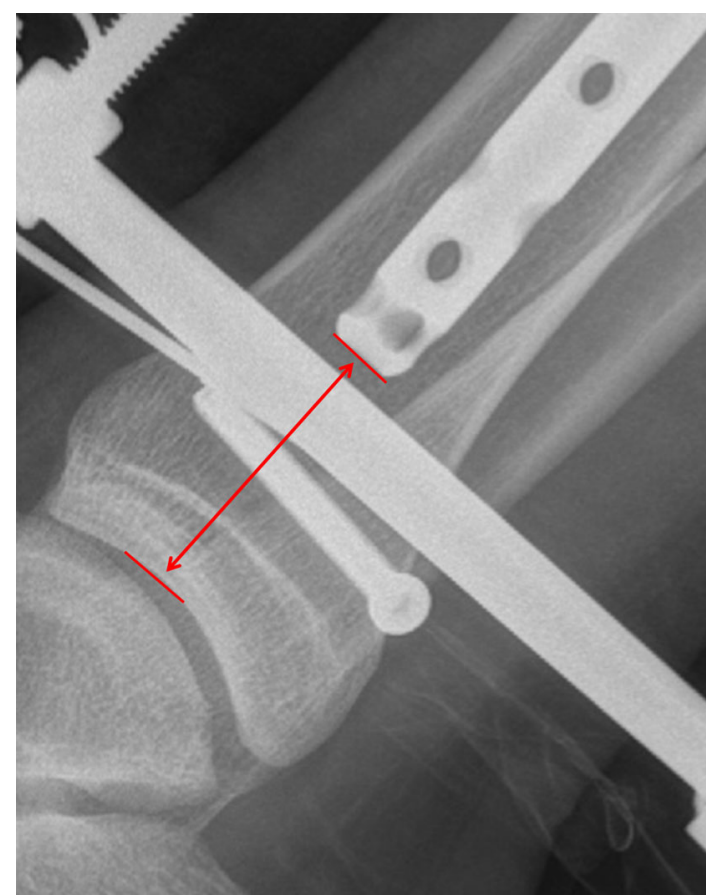

Fig. 7 A plain lateral radiograph taken immediately postoperatively of the distal tibia shows the distance (red double-sided arrow) from the distal tip of the nail to the tibial plafond.

paired t-test was used to find a difference in overall valgus deviation (change of mFTA), valgus deviation at proximal segments (change of Distance D), and valgus deviation at the distal segment (change of Angle A). Student's t-test and chi-square test were used to compare the preoperative variables and final length gain depending on the use of a blocking screw at the distal fragment. Association between overall valgus deviation and all possible factors were analyzed by a linear regression model. Multivariate analysis was performed with the explanatory variables, which 
were significant in the univariate analyses. A probability less than 0.05 was considered statistically significant. The statistical software MedCalc ${ }^{\circledR}$ (Version 11.6; MedCalc ${ }^{\circledR}$ Software, Mariakerke, Belgium) and R (Version 3.1.0; Comprehensive R Archive Network, GNU General Public

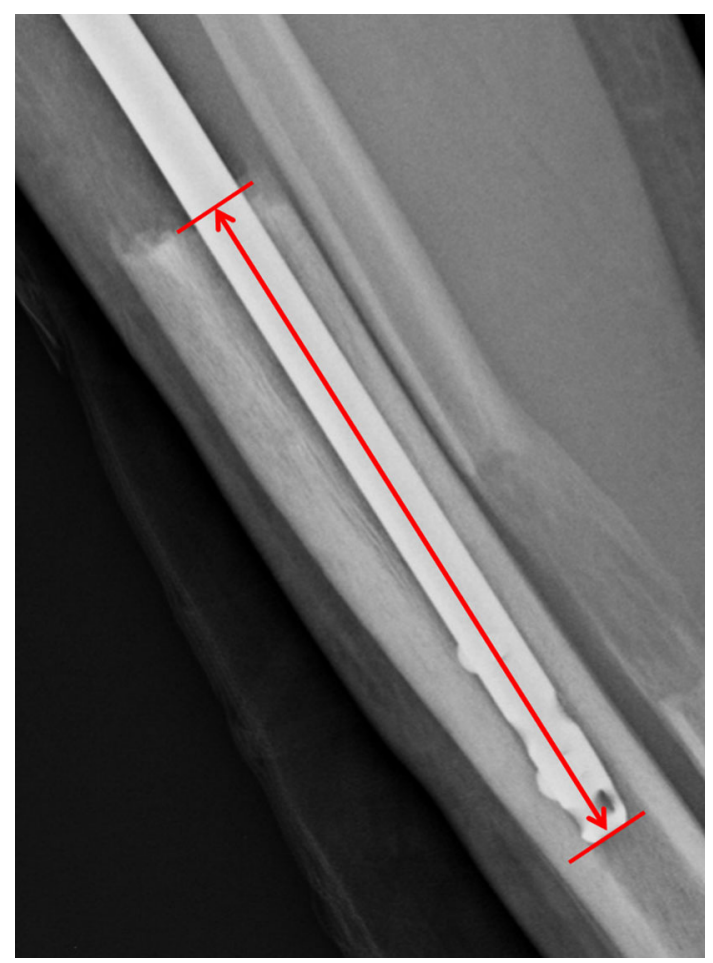

Fig. 8 A plain lateral radiograph of the tibia shows the length (red double-sided arrow) of the intramedullary nail in the distal segment after completion of distraction.
License, Boston, MA, USA) were used for all statistical analyses.

\section{Results}

The overall valgus deviation increased during lengthening of the tibia with the LON technique. The MPTA increased from $86^{\circ}\left(95 \% \mathrm{CI}, 85^{\circ}-86^{\circ}\right)$ preoperatively to $90^{\circ}(95 \% \mathrm{CI}$, $\left.89^{\circ}-91^{\circ}\right)(\mathrm{p}<0.001)$ after completion of lengthening. Similarly, the mFTA changed from $2.2^{\circ}$ varus $(95 \% \mathrm{CI}$, $3^{\circ}-1.4^{\circ}$ varus) to $2.6^{\circ}$ valgus (95\% $\mathrm{CI}, 1.8^{\circ}-3.4^{\circ}$ valgus) $(\mathrm{p}<0.001)$. There was no difference between preoperative MPTA and postoperative MPTA (Table 3). The valgus deviation increased during lengthening of the tibia at the proximal segment. The pairwise difference of Distance D between the immediate postoperative and final radiographs was $8 \pm 10 \mathrm{~mm}(\mathrm{p}<0.001)$. The valgus deviation increased at the distal segment as well during lengthening of the tibia. The pairwise difference of Angle A between the immediate postoperative and final radiographs was $1.0^{\circ} \pm 1.0^{\circ}(\mathrm{p}<0.001)$ (Table 3$)$.

Multivariate analysis of overall valgus deviation using a linear regression model showed that the use of a blocking screw was the only factor associated with a reduction in the overall valgus deviation (final mFTA minus preoperative mFTA) $(-2.62 ; 95 \% \mathrm{CI},-4.65$ to $-0.59 ; \mathrm{p}=0.013)$ (Table 4). The other factors (proximal fixation method, nail diameter and length, depth of nail insertion, length of the nail residing in the distal fragment at final, final length gain, and BMI) showed no effect on overall valgus deviation.

Table 3. Degree of valgus change in lengthening over nail of the tibia

\begin{tabular}{lll}
\hline Valgus change & 60 tibias & $95 \%$ CI value \\
\hline Valgus deviation overall (degrees) & & $85-86$ \\
Preoperative MPTA & $86 \pm 2$ & $85-86$ \\
Postoperative MPTA & $86 \pm 2$ & $89-91$ \\
Final MPTA & $90 \pm 2$ & -0.1 to 0.7 \\
Postoperative MPTA - preoperative MPTA & $0.3 \pm 1.2$ & $3.5-5$ \\
Final MPTA - preoperative MPTA & $4.2 \pm 2.5$ & $3-1.4$ varus \\
Preoperative mFTA & $2.2 \pm 2.5$ varus & $1.8-3.4$ valgus \\
Final mFTA & $2.6 \pm 2.7$ valgus & $3.9-5.7$ \\
Final mFTA - preoperative mFTA & $4.8 \pm 3.0$ & $<.142$ \\
Valgus deviation at proximal segment* (mm) & $-8 \pm 10$ & 6.001 \\
Distance D at final - Distance D at immediate postoperative & & $<0.001$ \\
Valgus deviation at distal segment ${ }^{*}$ (degrees) & $1 \pm 1$ & $0.8-1.2$ \\
Angle A at final - Angle A at immediate postoperative & $<0.001$ \\
\hline
\end{tabular}

Values are mean $\pm \mathrm{SD} ; *$ Distance $\mathrm{D}=$ distance between lateral margin of the intramedullary nail and inner margin of the lateral cortex at the proximal fragment; ${ }^{\dagger}$ Angle $\mathrm{A}=$ angle between the longitudinal axis of the intramedullary nail and longitudinal axis of the distal segment; MPTA $=$ medial proximal tibial angle; $\mathrm{mFTA}=$ mechanical femorotibial angle. 
Table 4. Multivariate results for overall valgus deviation (final mFTA - preoperative mFTA) using linear regression model

\begin{tabular}{|c|c|c|c|}
\hline Factors & Coefficient & $95 \% \mathrm{CI}$ & $\mathrm{p}$ value \\
\hline \multicolumn{4}{|l|}{ Proximal fixation method } \\
\hline Two ASLS screws* & 2.41 & -0.40 to 5.23 & 0.091 \\
\hline One ASLS screw and two oblique screws* & 3.18 & -0.29 to 6.64 & 0.071 \\
\hline Presence of blocking screw & -2.62 & -4.65 to -0.59 & 0.013 \\
\hline Diameter of nail & 0.47 & -0.64 to 1.57 & 0.399 \\
\hline Length of nail & -24.89 & -63.60 to 13.82 & 0.201 \\
\hline Depth of nail insertion ${ }^{\dagger}$ & 0.03 & -0.09 to 0.14 & 0.626 \\
\hline Length of the nail in the distal segment & 0.06 & -0.23 to 0.35 & 0.681 \\
\hline Final length gain & 0.07 & -0.07 to 0.21 & 0.328 \\
\hline BMI & 0.25 & -0.24 to 0.74 & 0.309 \\
\hline
\end{tabular}

* Two standard locking screws is the reference group; ${ }^{\dagger}$ depth of intramedullary nail insertion (mm) indicates the distance from the distal tip of the nail to the tibial plafond (immediate postoperative lateral radiograph); $\mathrm{mFTA}=$ mechanical femorotibial angle.

\section{Discussion}

During tibial lengthening, soft tissues of the posterolateral compartment produce distraction-resisting forces causing valgus and anterior angulation [1]. Development of such deformities is debated with the LON technique because the intramedullary nail is thought to resist deforming forces and maintain alignment of the distracted bone; however, the degree to which this is true is not well described. In the current study, we observed an increase in valgus deviation during tibial lengthening with the LON technique. The valgus deviation was evident in the proximal (valgus deviation of the intramedullary nail in relation to the proximal fragment) and distal segments (valgus deviation of the distal fragment in relation to the intramedullary nail). Use of a blocking screw was the only factor that we examined that was associated with decreased valgus deviation, whereas the other factors were not, including the use of ASLS screws in proximal fixation, use of a thicker and longer intramedullary nail, deeper insertion of the intramedullary nail during surgery, longer length of the nail residing in the distal fragment after completion of lengthening, shorter final length gain, and lower BMI.

Several limitations should be mentioned. First, axial deviation such as valgus depends on the entry point and direction of the intramedullary nail. During surgery, if the entry point is made at the medial aspect of the proximal tibia and directed laterally inside the medullary canal, it causes intentional valgus deviation. However, because there was no significant difference between preoperative MPTA and postoperative MPTA, we presumed that the valgus deviation occurred during distraction, not during surgery. Second, two different methods were used to show the valgus deviation at the proximal and distal segments using Distance D (mm) and Angle A (degrees), respectively. Because a direct comparison of these two methods cannot be done, we are unable to determine in which segment the valgus deviation occurred more. However, we believe that each method is the best indicator reflecting the degree of valgus deviation at each segment with reduced measurement variability. Third, a small sample size can have a large type II error for statistical tests. Sixty tibias were used to find the factors associated with decreased valgus deviation among eight possible surgical and patient variables. Although statistical power analysis cannot determine if this sample size is large enough at this setting, this could be a relatively small number. Finally, this study was based on a retrospective review, and use of a blocking screw was not randomized. Even though there was no statistical difference in preoperative variables between the two groups (one group with and one without a blocking screw) (Table 2), selection bias factors might have influenced the results.

In the classic Ilizarov method, axial deviation such as valgus has been known to develop during tibial lengthening [1]. However, the concomitant use of an intramedullary nail, such as with tibial LON, can act as a strong resistance to the deforming forces, minimizing axial deviations compared with the classic Ilizarov method. Some studies $[2,3,15,20,21]$ compared the classic Ilizarov method with the LON technique and showed that axial deviation occurred in $4 \%$ to $13 \%$ of tibias with the classic Ilizarov method, whereas it was $0 \%$ to $2 \%$ with the LON technique. However, Kim et al. [8] reported that they observed 20 segments $(14 \%)$ that showed valgus angulation with a mean of $10^{\circ}$ using the LON technique. Shyam et al. [17] reported that they observed valgus angulation with a mean of $10^{\circ}$ with the classic Ilizarov method, whereas it was $7^{\circ}$ 
with the LON technique. In the current study, we observed a mean valgus shift of $4.2^{\circ}$ in the MPTA and $4.8^{\circ}$ in the mFTA. We also saw an increase in valgus deviation at the proximal and distal segments, and use of a blocking screw was the only effective method to decrease valgus deviation.

Since being introduced by Krettek et al. [10, 11], a blocking screw has been established as an important adjunct for intramedullary nailing and fracture reduction [4]. It can direct the intramedullary nail, control angular deformity, and increase stability of the bone-nail construct [4, 16, 19]. For tibial lengthening with the LON technique, the distraction-resisting force causes angulation of the nail in a limited medullary space, resulting in valgus deviation (Fig. 9). In addition, the angulation may be caused by instability that results from the difference between the diameters of the nail and medullary cavity. A blocking screw also can be effective in distraction osteogenesis if the intramedullary nail is used in lengthening. By narrowing the medullary canal, it allows only a limited space for the intramedullary nail to deviate as the distraction progresses. In the current study, placement of a blocking screw had a highly significant association with reduction of valgus deviation (coefficient $=-2.62 ; \mathrm{p}=0.013$ ) in multivariate analysis and was the only factor we identified that was associated with reduced valgus deviation when the screw was inserted just below the osteotomy level and just lateral to the nail in the distal segment.

However, use of blocking screws does not necessarily mean that it can prevent valgus deviation during LON of the tibia. It is essential that it be placed at the right position to function properly. Accurate placement is important to obtain its maximum benefit. A blocking screw can be placed in the proximal segment and the distal segment. We assumed that valgus deviation of the proximal segments could be prevented by increasing stability of the proximal locking screws, and this is why we switched the fixation method of the proximal segment during the study period. Therefore, we inserted a blocking screw in only the distal segment. However, valgus deviation occurred at the proximal segment without a blocking screw. We subsequently changed our practice to using proximal and distal blocking screws. Additional studies should seek to evaluate whether an additional blocking screw in the proximal segment might be more effective. In addition, impingement between the nail and the blocking screw is a potential concern, making the bone difficult to be distracted. However, we did not experience this phenomenon. No patients with a blocking screw experienced complications during surgery or during the lengthening period. No complications, such as impingement or screw breakage, were observed during nail removal in 23 patients $(82 \%)$ with blocking screws; the remaining five patients have not yet undergone nail removal.

We found that valgus deviation does occur during tibial lengthening using the LON technique, but placement of the blocking screw might help minimize the likelihood that severe valgus will occur. Future prospective studies should seek to confirm this preliminary finding.
Fig. 9A-C Plastic models of the osteotomized tibia, which was lengthened with an intramedullary nail, show the valgus deviation during the tibial lengthening over an intramedullary nail. (A) Without a blocking screw, a considerable amount of valgus deviation occurred at the proximal and distal segments. (B) With a blocking screw at the distal fragment near the osteotomy level, overall valgus deviation decreased with almost no valgus at the distal fragment. (C) With two blocking screws at the proximal and distal fragments, no valgus deformity developed. The rubber band at the lateral aspect is for soft tissue resistance.
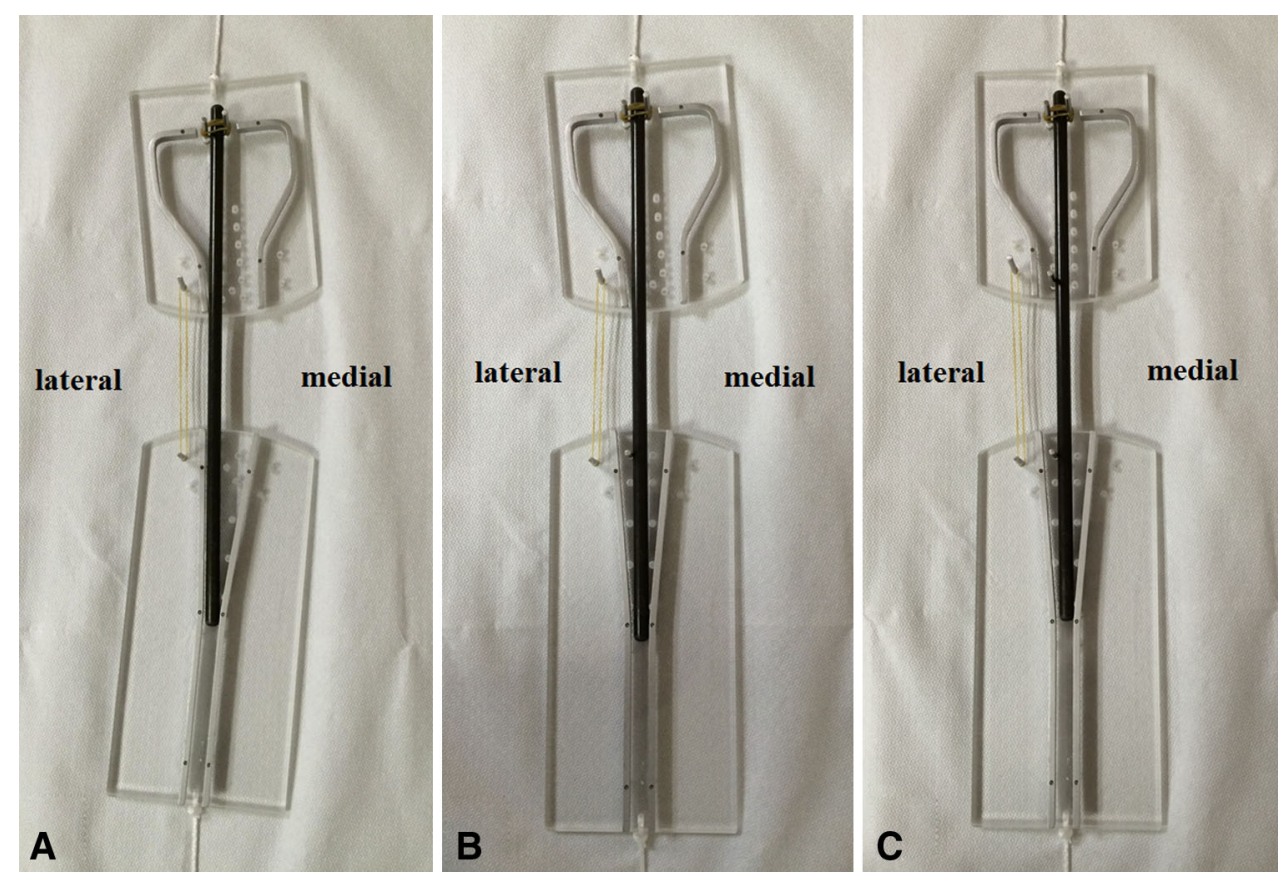
Acknowledgments We thank Hakan Kinik MD (Department of Orthopaedics and Traumatology, Ankara University, School of Medicine, Ankara, Turkey), Oguz Poyanli, MD (Department of Orthopedics and Traumatology, Medeniyet University Goztepe Training and Research Hospital, Istanbul, Turkey), and Hans-Werner Stedtfeld, MD, PhD (Department of Trauma, Klinikum Nurnberg Klinikum Sud, Nurnberg, Germany) for providing the plastic model that showed the valgus deviation during the tibial lengthening over the intramedullary nail shown in Figure 9, and Jung Ho Park (Physical Therapist, Department of Rehabilitation, CHA Bundang Medical Center, CHA University) for his enthusiastic and cooperative work for our patients.

\section{References}

1. Catagni M. Lengthening of the tibia. In: Maiocchi A, Aronson J, Association for the Study and Application of Ilizarov's Method, eds. Operative Principles of Ilizarov: Fracture Treatment, Nonunion, Osteomyelitis, Lengthening, Deformity Correction. Baltimore, MD: Williams \& Wilkins; 1991:288-309.

2. El-Husseini TF, Ghaly NA, Mahran MA, Al Kersh MA, Emara KM. Comparison between lengthening over nail and conventional Ilizarov lengthening: a prospective randomized clinical study. Strategies Trauma Limb Reconstr. 2013;8:97-101.

3. Guo Q, Zhang T, Zheng Y, Feng S, Ma X, Zhao F. Tibial lengthening over an intramedullary nail in patients with short stature or leg-length discrepancy: a comparative study. Int Orthop. 2012;36:179-184.

4. Hannah A, Aboelmagd T, Yip G, Hull P. A novel technique for accurate Poller (blocking) screw placement. Injury. 2014;45: 1011-1014.

5. Herzenberg JE, Paley D. Tibial lengthening over nails (LON). Tech Orthop. 1997;12:250-259.

6. Jain S, Harwood P. Does the use of an intramedullary nail alter the duration of external fixation and rate of consolidation in tibial lengthening procedures? A systematic review. Strategies Trauma Limb Reconstr. 2012;7:113-121.

7. Kim SJ, Cielo Balce G, Huh YJ, Song SY, Song HR, Kim SJ. Deep intramedullary infection in tibial lengthening over an intramedullary nail. Acta Orthop Belg. 2011;77:506-515.

8. Kim SJ, Mandar A, Song SH, Song HR. Pitfalls of lengthening over an intramedullary nail in tibia: a consecutive case series. Arch Orthop Trauma Surg. 2012;132:185-191.
9. Kocaoglu M, Eralp L, Kilicoglu O, Burc H, Cakmak M. Complications encountered during lengthening over an intramedullary nail. J Bone Joint Surg Am. 2004;86:2406-2411.

10. Krettek C, Miclau T, Schandelmaier P, Stephan C, Mohlmann U, Tscherne H. The mechanical effect of blocking screws ('Poller screws') in stabilizing tibia fractures with short proximal or distal fragments after insertion of small-diameter intramedullary nails. J Orthop Trauma. 1999;13:550-553.

11. Krettek C, Stephan C, Schandelmaier P, Richter M, Pape HC, Miclau T. The use of Poller screws as blocking screws in stabilising tibial fractures treated with small diameter intramedullary nails. J Bone Joint Surg Br. 1999;81:963-968.

12. Lee DH, Ryu KJ, Shin DE, Kim HW. Botulinum toxin a does not decrease calf pain or improve ROM during limb lengthening: a randomized trial. Clin Orthop Relat Res. 2014;472:3835-3841.

13. Lee DH, Ryu KJ, Song SH, Park KW, Song HR. Clinical implication of sagittal translation in lengthening over nail of tibia. Eur J Orthop Surg Traumatol. 2014;24:1579-1585.

14. Paley D. Problems, obstacles, and complications of limb lengthening by the Ilizarov technique. Clin Orthop Relat Res. 1990;250:81-104.

15. Park HW, Yang KH, Lee KS, Joo SY, Kwak YH, Kim HW. Tibial lengthening over an intramedullary nail with use of the Ilizarov external fixator for idiopathic short stature. J Bone Joint Surg Am. 2008;90:1970-1978.

16. Shahulhameed A, Roberts CS, Ojike NI. Technique for precise placement of Poller screws with intramedullary nailing of metaphyseal fractures of the femur and the tibia. Injury. 2011;42:136-139.

17. Shyam AK, Song HR, An H, Isaac D, Shetty GM, Lee SH. The effect of distraction-resisting forces on the tibia during distraction osteogenesis. J Bone Joint Surg Am. 2009;91:1671-1682.

18. Simpson AH, Cole AS, Kenwright J. Leg lengthening over an intramedullary nail. J Bone Joint Surg Br. 1999;81:1041-1045.

19. Stedtfeld HW, Mittlmeier T, Landgraf T, Ewert A. The logic and clinical applications of blocking screws. J Bone Joint Surg Am. 2004;86(suppl 2):17-25.

20. Sun XT, Easwar TR, Manesh S, Ryu JH, Song SH, Kim SJ, Song HR. Complications and outcome of tibial lengthening using the Ilizarov method with or without a supplementary intramedullary nail: a case-matched comparative study. J Bone Joint Surg Br. 2011;93:782-787.

21. Watanabe K, Tsuchiya H, Sakurakichi K, Yamamoto N, Kabata $\mathrm{T}$, Tomita K. Tibial lengthening over an intramedullary nail. J Orthop Sci. 2005;10:480-485. 